\title{
有机电解质在胶束催化聚苯乙烯氯甲基化反应中的作用
}

\author{
高保娇*＼cjkstart张＼cjkstart昕＼cjkstart刘启发 \\ (中北大学化学工程系, 太原 030051)
}

\begin{abstract}
摘要 在实施聚苯乙烯氯甲基化反应的胶束催化体系中加人四丁基溴化铵 ((Bu) $\left.{ }_{4} \mathrm{NBr}, \mathrm{TBAB}\right)$, 研究了有机电解 质 TBAB 对胶束催化反应的影响规律. 实验结果表明, 在非离子表面活性剂 NP-10 及阴离子表面活性剂 SDS 的 胶束催化体系中, $\mathrm{TBAB}$ 的加人使聚苯乙烯氯甲基化反应的速率明显增大, 前者尤为突出; 而在阳离子表面活性 剂 $\mathrm{CTAB}$ 的胶束催化体系中, TBAB 的加人几乎对反应速率无促进作用. 这种结果一方面归因于加人电解质 TBAB 会降低 SDS 的临界胶束浓度, 从而增强对聚苯乙烯四氯化碳溶液的增溶能力; 更主要的原因是 TBAB 的 丁基与表面活性剂碳氢链间的疏水相互作用会使季铵离子 $(\mathrm{Bu})_{4} \mathrm{~N}^{+}$嵌人 SDS 的胶束之中, 结合到 NP-10 的胶束 表面, 使 SDS 胶束的阴离子头基对亲核取代反应(控制步骤)的禁阻作用得以减缓, 使 NP-10 的胶束表面携带了 正电荷, 显著促进亲核取代反应的进行, 而对于 $\mathrm{CTAB}$ 的胶束, 由于静电排斥作用, 季铵离子 $(\mathrm{Bu})_{4} \mathrm{~N}^{+}$不能接近 $\mathrm{CTAB}$ 的胶束, 故 TBAB 的加人对聚苯乙烯氯甲基化反应不产生作用.
\end{abstract}

关键词：胶束催化，表面活性剂，有机电解质，疏水相互作用，聚苯乙烯，氯甲基化 中图分类号：0647

\section{Effect of Organic Electrolyte on Chloromethylation of Polystyrene in Micellar Catalytic System}

\author{
GAO, Bao-Jiao* $\quad$ ZHANG, Xin $\quad$ LIU, Qi-Fa \\ (Chemical Engineering Department, North University of China, Taiyuan 030051, P. R. China)
}

\begin{abstract}
Organic electrolyte tetrabutyl ammonium bromide $\left((\mathrm{Bu})_{4} \mathrm{NBr}, \mathrm{TBAB}\right)$ was added into micellar catalysis system in which chloromethylation reaction of polystyrene was carried out, and the effect of TBAB on the catalytic reaction was investigated. The experiment results show that the adding of TBAB speeds up obviously the chloromethylation reaction of polystyrene in the micellar catalytic systems in which nonionic surfactant NP-10 and anionic surfactant SDS are used, especially in the system in which non-ionic surfactant NP-10 is used the acceleration is more distinct. On the contrary, in the micellar catalytic systems in which cationic surfactant CTAB is used, TBAB hardly influences the reaction rate. On one hand the reason for this may attribute to the decrease of critical micelle concentration of SDS and the increase of solubilization of polystyrene in the micellar solution because of adding electrolyte $\mathrm{TBAB}$, on the other hand, a more primary reason is that organic cation $(\mathrm{Bu})_{4} \mathrm{~N}^{+}$of TBAB may be embedded into micelles due to the synergistic effect of electrostatic attraction and hydrophobic interaction between butyl groups and the hydrocarbon chains of SDS, so that the inhibiting effect of anionic head groups of SDS for the nucleophilic substitution reaction that is a controlling step is weakened. Under hydrophobic interaction, organic cation $(\mathrm{Bu})_{4} \mathrm{~N}^{+}$of TBAB may also be combined on the surface of NP-10 micelles, so that the surface of NP-10 micelles charged positively accelerates distinctly the nucleophilic substitution reaction. For CTAB system, the cation $(\mathrm{Bu})_{4} \mathrm{~N}^{+}$can not be closed to the micelles of $\mathrm{CTAB}$ owing to electrostatic repellence, so the adding of TBAB can not speed up the rate of chloromethylation reaction of polystyrene.
\end{abstract}

Received:May 23, 2005; Revised: June 21, 2005. Correspondent, E-mail: gaobaojiao@126.com; Tel:0351-3921414

(C) Editorial office of Acta Physico-Chimica Sinica 
Keywords: Micellar catalysis, Surfactant, Organic electrolyte, Hydrophobic interaction, Polystyrene, Chloromethylation

胶束催化是促进油-水两相反应物进行有机反 应的有效手段, 借助于表面活性剂的作用, 油相反应 物以微胶束(增溶于胶束中)的形式分散于水介质中, 极大地扩大了油相反应物与水相中进攻试剂的接触 界面, 而且在静电效应、局部浓集效应等作用下 ${ }^{[1-3]}$, 油-水两相间的反应速率会被显著加速, 而且可使反 应条件变得更加温和, 提高有机合成的效率, 目前胶 束催化已用于多类有机合成 ${ }^{[1-8]}$.

氯甲基化的聚苯乙烯(chloromethylated polystyrene, CMPS)为线型聚苯乙烯(polystyrene, PSt)分子 链中苯环对位的氢原子部分或全部被氯甲基取代后 的产物. 由于氯甲基具有很高的化学活性, 因此氯甲 基化的聚苯乙烯是多种功能高分子化合物的前驱 体, 比如, 以 CMPS 为原子转移自由基聚合(ATRP) 的大分子引发剂, 可合成接枝、树枝状及高枝化星 状共聚物 ${ }^{[91}$; 可合成带有特殊官能团的离子交换树 脂、金属离子的鳌合树脂及有机物的吸附树脂 ${ }^{[10]}$; 如 果再经后交联, 可制备功能高分子微球, 用于生物大 分子分离、纯化、固定化酶及色谱固定相等 ${ }^{[11]}$, 显然, 氯甲基化的聚苯乙烯在众多科技领域都将具有广阔 的应用前景. 以对氯甲基苯乙烯 $(p$-chloromethylstyrene, CMS)为单体进行均聚合或与苯乙烯共聚合, 这是目 前制备氯甲基化聚苯乙烯的常用方法 ${ }^{[10-11]}$, 但由于 CMS 价格高, 该方法显然是不经济的. 能否开拓出 聚苯乙烯氯甲基化的新方法呢? 能否采用胶束催化 的方法(类似于小分子的有机反应), 使线性大分子 聚苯乙烯直接得以氯甲基化? 前文报道了采用胶束 催化的方法, 高效率地实现了聚苯乙烯的氯甲基 化 ${ }^{[12]}$ : 将聚苯乙烯溶于四氯化碳中, 此油相增溶于离 子表面活性剂的胶束中, 与水介质中的甲醛及盐酸 较快速进行反应, 实现了聚苯乙烯氯甲基化反应. 在 此基础上本文进行了进一步研究, 在胶束催化体系 中加人有机电解质四丁基溴化铵(TBAB), 四丁基溴 化铵的丁基与表面活性剂碳氢链间的疏水相互作 用, 使得季铵离子 $(\mathrm{Bu})_{4} \mathrm{~N}^{+}$可嵌人阴离子表面活性剂 十二烷基硫酸钠(SDS)的胶束之中, 结合至非离子 表面活性剂壬基酚聚氧乙烯醚-10(NP-10)的胶束表 面 ${ }^{[12]}$, 明显促进了亲核取代反应(氯甲基化反应的控 制步骤), 显著加快了聚苯乙烯氯甲基化反应的速 率. 本研究的目的是为采用胶束催化实现线型大分
子的化学反应提供相应的理论依据.

\section{1 实验部分}

\section{1 试剂与仪器}

聚苯乙烯(PSt, 北京燕山石化公司); 十六烷基 三甲基溴化铵 (CTAB, 北京化学试剂研究所), 化 学纯; 十二烷基硫酸钠(SDS, 北京中联化工试剂厂), 分析纯; 壬基酚聚氧乙烯醚-10(NP-10, 开封化学试 剂总厂), 化学纯;四丁基澳化铵(tetrabutyl ammonium bromide, TBAB, 天津市光复精细化工研究所), 分析 纯; 四氯化碳(石家庄有机化工厂), 分析纯; 甲醛 (37\% 40\%水溶液, 石家庄有机化工厂), 分析纯; 浓 盐酸(天津化学试剂六厂), 分析纯;三氯化磷(天津滨 海化工厂), 化学纯.

瑞士 Perkin-Elmer1700 型傅立叶红外光谱仪; 上 海尤尼柯公司 UV-2602 分光光度计;湖南长沙仪器厂 WGR-1 氧弹式量热计; 天津奥特公司 AS20500ADT 超声波浴槽; 承德仪器厂JZHY-180 型界面张力仪.

\section{2 外加电解质后表面活性剂 $\mathrm{cmc}$ 的测定}

在表面活性剂水溶液中加人一定量的电解质, 使用表面张力仪(环法)在恒定温度下测定水溶液的 表面张力, 绘制水溶液的表面张力对表面活性剂浓 度曲线 $(\gamma-c$ 曲线), 确定临界胶束浓度 $\mathrm{cmc}$; 改变电 解质的浓度, 依上法确定表面活性剂在不同电解质 浓度下的临界胶束浓度 $\mathrm{cmc}$; 绘制表面活性剂临界 胶束浓度对电解质浓度的曲线 ( $\mathrm{cmc}-c$ 曲线), 考察外 加电解质对表面活性剂临界胶束浓度的影响. 为探索 有机电解质对表面活性剂胶束化行为的影响不同于 无机电解质的规律, 在表面活性剂水溶液中分别加人 有机电解质四丁基溴化铵与溴化钠进行上述测定.

\section{3 加入 TBAB 后胶束溶液对聚苯乙烯增溶性能} 的测定

配制一定浓度的溶有聚苯乙烯的四氯化碳溶液 及一定浓度的表面活性剂水溶液; 将足量的溶有聚 苯乙烯的四氯化碳溶液与表面活性剂溶液混合, 超 声波震荡 $20 \mathrm{~min}$, 静置, 至水层呈无色透明状, 水层 为增溶有聚苯乙烯(四氯化碳溶液)的表面活性剂胶 束溶液, 使用紫外分光光度法 ${ }^{[12]}$ 测定聚苯乙烯增溶 量, 并绘制聚苯乙烯增溶量-表面活性剂浓度关系 曲线. 配制一定浓度的含有四丁基溴化铵的表面活 
性剂水溶液, 依上法测定聚苯乙烯(四氯化碳溶液) 在其中的增溶量, 并绘制聚苯乙烯增溶量-表面活 性剂浓度关系曲线, 对两种曲线进行比较, 考察有机 电解质 TBAB 对聚苯乙烯在表面活性剂胶束溶液 中增溶量的影响规律.

\section{4 加入 TBAB 实施聚苯乙烯的的胶束催化氯甲 基化}

在装有搅拌器、滴液漏斗及冷凝管的 $250 \mathrm{~mL}$ 四口瓶中, 加人足量溶有聚苯乙烯的四氯化碳溶液 与适量的浓度一定的表面活性剂水溶液, 摚拌 $5 \mathrm{~h}$, 使聚苯乙烯的四氯化碳溶液充分增溶于表面活性剂 胶束溶液中. 加人一定体积的浓盐酸与甲醛溶液, 升 温至要求的温度, 摚拌下滴加三氯化磷, 一定时间后 结束反应, 用乙醇为沉淀剂, 将氯甲基化反应后的聚 苯乙烯沉淀出来, 用蒸馏水及乙醇洗至无氯离子后, 真空干燥, 采用氧弹燃烧-佛尔哈德法测定样品中的 氯含量 ${ }^{[12]}$, 并以聚苯乙烯中苯环四号全部氯甲基化 为标准, 计算聚苯乙烯样品的氯甲基化程度 $(\%)$.

为探索四丁基溴化铵在不同胶束催化体系中对 聚苯乙烯氯甲基化反应的影响规律, 分别在改变表 面活性剂类型, 改变四丁基溴化铵加人量等条件下, 按上述步骤进行实验.

\section{2 结果与讨论}

\section{1 有机电解质对表面活性剂胶束化行为的影响}

在阴离子表面活性剂 SDS 与阳离子表面活性 剂 CTAB 溶液中, 分别加人无机电解质 $\mathrm{NaBr}$ 与有 机电解质 $\mathrm{TBAB}$, 它们对这两种离子型表面活性剂 临界胶束浓度 $(\mathrm{cmc})$ 的影响规律分别示于图 1(a)与 图 l(b).

SDS 的临界胶束浓度为 $8.1 \mathrm{mmol} \cdot \mathrm{L}^{-1}\left(25^{\circ} \mathrm{C}\right)$. 从

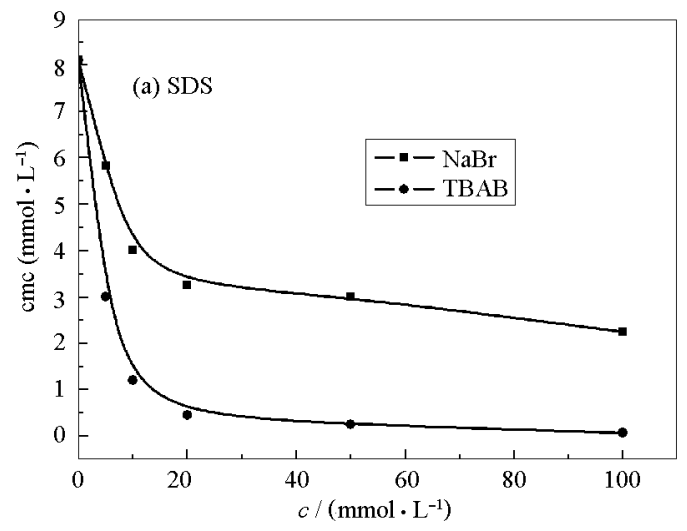

图 1(a)中看到, 随着少量电解质 $\mathrm{NaBr}$ 及 $\mathrm{TBAB}$ 的 加人, 阴离子表面活性剂 SDS 的临界胶束浓度 $(\mathrm{cmc})$ 急剧下降, 且后者的作用更为显著. 加人的 $\mathrm{NaBr}$, 在静电吸引下, 反离子 $\mathrm{Na}^{+}$会结合到 SDS 胶 束表面, 从而屏蔽胶束阴离子头基间的静电排斥力, 促进 SDS 的聚集 ${ }^{\left[{ }^{[3]}\right]}$, 故使其 $\mathrm{cmc}$ 下降. 从作为电解 质的角度分析, TBAB 的有机离子 $(\mathrm{Bu})_{4} \mathrm{~N}^{+}$同样具有 上述静电作用, 此外 $(\mathrm{Bu})_{4} \mathrm{~N}^{+}$的丁基还可能与 SDS 的 碳氢链之间发生疏水相互作用, 在静电吸引与疏水 相互作用两种作用的协同下, 有机正离子 $(\mathrm{Bu})_{4} \mathrm{~N}^{+}$会 更紧密地与胶束结合, 季铵离子 $(\mathrm{Bu})_{4} \mathrm{~N}^{+}$很可能被 嵌人 SDS 胶束之中 ${ }^{[1]]}$, 这就能更有效地屏蔽胶束阴 离子头基间的静电斥力, 故 TBAB 降低 SDS 临界胶 束浓度的能力更强.

可以推测, 对于非离子表面活性剂 NP-10 胶束 溶液, 加人有机电解质 $\mathrm{TBAB}$, 虽然胶束头基无负电 性, $(\mathrm{Bu})_{4} \mathrm{~N}^{+}$与胶束之间无静电吸引力, 但 $(\mathrm{Bu})_{4} \mathrm{~N}^{+}$的 丁基与 NP-10 的碳氢链之间, 也会产生疏水相互作 用, 有机正离子 $(\mathrm{Bu})_{4} \mathrm{~N}^{+}$也会被结合到胶束表面.

$\mathrm{CTAB}$ 临界胶束浓度为 $0.92 \mathrm{mmol} \cdot \mathrm{L}^{-1}\left(25^{\circ} \mathrm{C}\right)$. 从图 1(b)中看到, 在阳离子表面活性剂 CTAB 胶束 溶液中, 无机电解质 $\mathrm{NaBr}$ 与有机电解质 $\mathrm{TBAB}$ 对 $\mathrm{CTAB}$ 胶束化行为的影响是一致的, 只要二者的浓 度相同, 降低 CTAB 临界胶束浓度的能力就相同. 这是因为 $\mathrm{NaBr}$ 与 $\mathrm{TBAB}$ 提供了相同的反离子 $\mathrm{Br}^{-}$, 而由于静电斥力, $\mathrm{TBAB}$ 的有机正离子 $(\mathrm{Bu})_{4} \mathrm{~N}^{+}$不能 接近 CTAB 的胶束, 不可能产生疏水相互作用, 故 $\mathrm{NaBr}$ 与 $\mathrm{TBAB}$ 影响 $\mathrm{CTAB}$ 胶束化行为的因素是相 同的, 只有反离子 $\mathrm{Br}^{-}$对胶束阳离子头基间静电斥 力的屏蔽作用, 故有上述规律.

此外, 有机电解质对表面活性剂胶束化行为的

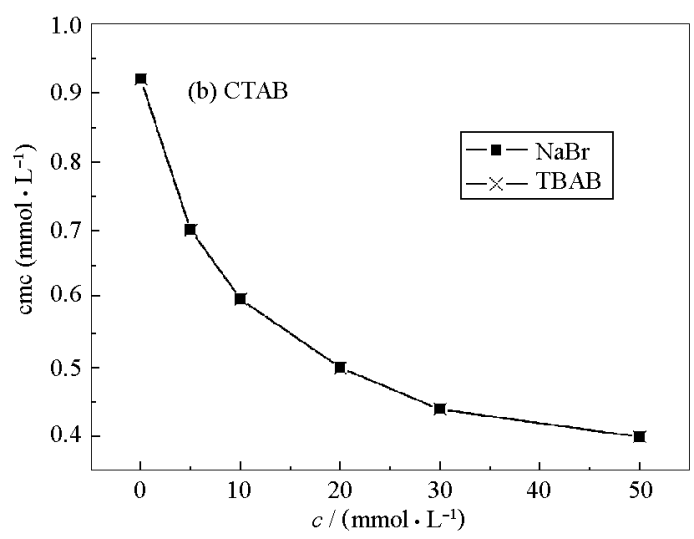

图 1 外加电解质的浓度与离子型表面活性剂临界胶束浓度的关系

Fig.1 Effect of adding electrolyte on the critical micelle concentrations of ionic surfactants 

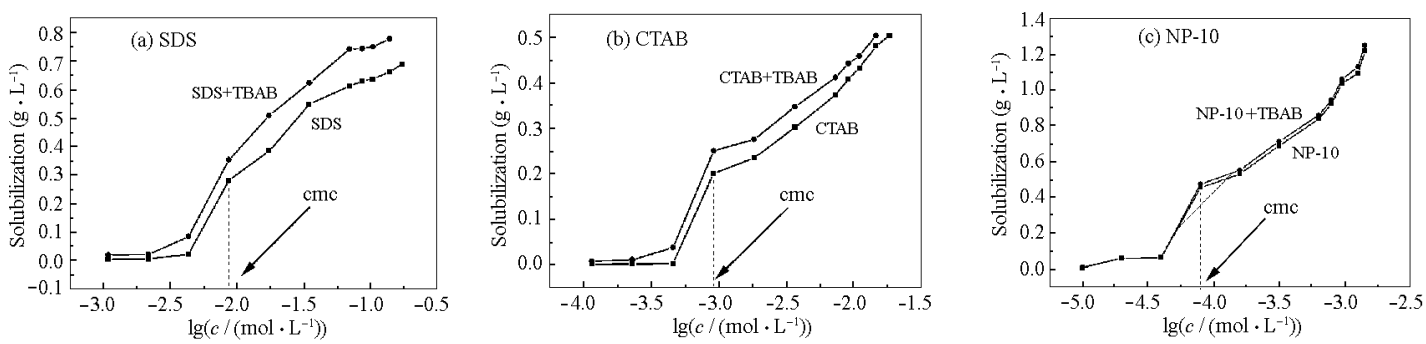

图 2 外加有机电解质对表面活性剂胶束溶液增溶聚苯乙烯能力的影响

Fig.2 Solubilization of polystyrene in aqueous surfactant solutions in the presence or absence of TBAB

影响还会反映在聚集数 $N_{\text {agg }}$ 的改变, 电解质 $\mathrm{NaBr}$ 及 $\mathrm{TBAB}$ 的加人都会使离子型表面活性剂 SDS 与 $\mathrm{CTAB}$ 的 $N_{\text {agg }}$ 增大 ${ }^{[13]}$, 但依据上述分析, 可以预测, 有机电解质 TBAB 对 SDS 的 $N_{\mathrm{agg}}$ 影响将可能大于 对 CTAB 的影响, 需要进一步研究.

\subsection{TBAB 对聚苯乙烯增溶量的影响}

$15{ }^{\circ} \mathrm{C}$ 下聚苯乙烯在各种胶束溶液中的增溶曲 线如图 2 所示. 从图 2 中看到无论是阳离子、阴离子 还是非离子表面活性剂, 当浓度处于临界胶束浓度 $(\mathrm{cmc})$ 以下时, 它们的水溶液几乎不溶解聚苯乙烯; 一旦它们的浓度达到临界浓度, 线型聚苯乙烯便 会在胶束溶液中发生增溶, 而且随着表面活性剂浓 度的增大, 增溶量不断增大. 线型聚苯乙烯在表面 活性剂胶束溶液中的增溶机理为, 当表面活性剂在 水溶液中形成胶束后, 有机溶剂四氯化碳便会增溶 其中, 伴随着四氯化碳的增溶, 溶解在四氯化碳中的 线型聚苯乙烯也会转移至胶束之中 ${ }^{[12]}$. 图 2 中还显 示出非离子表面活性剂 NP-10 对聚苯乙烯的增溶 能力远高于离子型表面活性剂 CTAB 与 SDS, 其原 因在于两点: 其一非离子表面活性剂的 $\mathrm{cmc}$ 较小, 聚集数 $N_{\mathrm{agg}}$ 较大, 故增溶能力较强; 其二非离子表 面活性剂分子在胶团中的排列较离子型表面活性剂 分子在胶团中的排列更为松散, 因而在不破坏原有 结构的条件下能容纳较多的外加分子, 即增溶能力 较强. 从图 2 中还看到, 在离子型表面活性剂水溶液 中加人电解质 $\mathrm{TBAB}$ 后, 胶束溶液对聚苯乙烯的增 溶能力均增强. 如 2.1 节分析, 电解质 $\mathrm{TBAB}$ 的加 人, 会降低离子型表面活性剂的 $\mathrm{cmc}$, 并可能增大表 面活性剂的聚集数 $N_{\text {agg, }}$, 前者使一定浓度的胶束溶 液中胶束数量增多, 后者则使胶束内核增大, 两者的 变化均会有利于碳氢化合物的增溶, 故两种离子型 表面活性剂胶束溶液对聚苯乙烯的增溶能力均有明 显的增强.

但向非离子型表面活性剂 NP-10 溶液中加人
电解质 TBAB 后, 虽然亦会使聚苯乙烯的增溶能力 有所增强, 但增溶量的改变很小. 这是由于外加电解 质对非离子型表面活性剂聚集数 $N_{\text {agg }}$ 影响不大, 且 对其 $\mathrm{cmc}$ 影响也较小, 只有电解质的浓度较高时才 会产生可觉察的效应 ${ }^{[13]}$.

\section{3 在外加有机电解质的胶束催化体系中实现聚 苯乙烯的氯甲基化}

2.3.1氯甲基化反应机理与表面活性剂的类型对氯甲基 化反应的影响

图 3 表示出 SDS、CTAB、NP-10 三种表面活性 剂对聚苯乙烯氯甲基化的胶束催化作用. 当表面活 性剂浓度很低时, 聚苯乙烯氯甲基化的程度很低, 而 且不随浓度变化; 而当表面活性剂浓度达到其临界 胶束浓度时, 聚苯乙烯氯甲基化程度陡然升高, 而且 随浓度增大而继续升高; 当浓度达到 $10 \mathrm{cmc}$ 时(离 子型表面活性剂), 氯甲基化程度变化变得很缓慢. 图 3 充分显示出线型聚苯乙烯在胶束催化下高效率 地发生了苯环的氯甲基化反应. 当表面活性剂在水 溶液中形成胶束后, 聚苯乙烯会伴随四氯化碳增溶 于胶束之中, 油/水两相界面迅速增大, 加快了界面

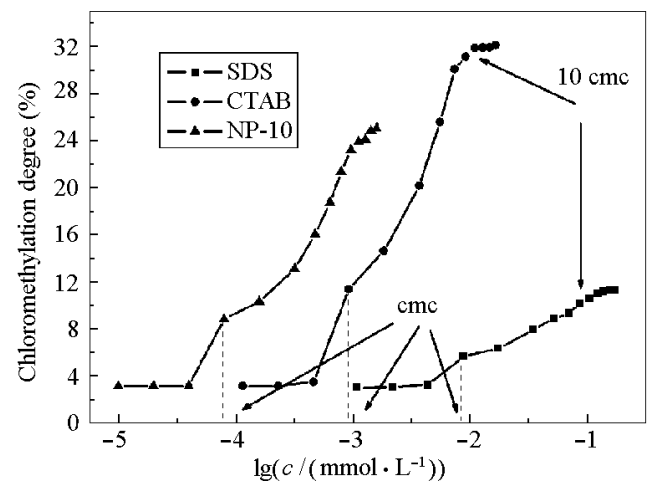

图 3 聚苯乙烯氯甲基化程度随表面活性剂浓度的变化

Fig.3 Variation of chloromethylation of PSt with surfactant concentration for three types of surfacatants

reaction temperature: $65{ }^{\circ} \mathrm{C}$; reaction time: $4 \mathrm{~h}$ 


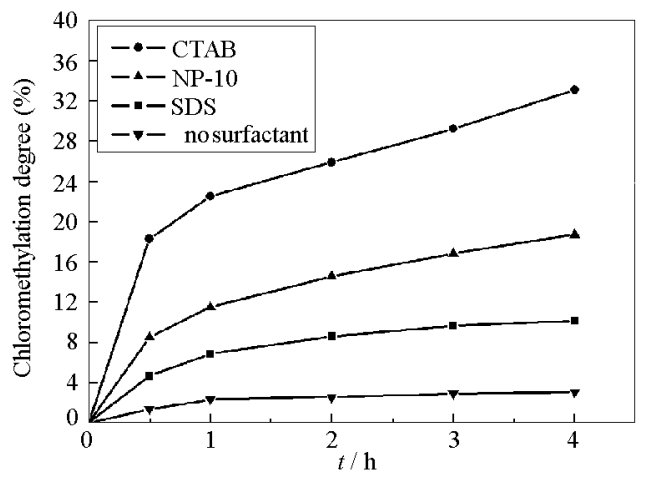

图 4 聚苯乙烯氯甲基化程度随时间的变化

Fig.4 Variation of chloromethylation of PSt with reaction time for three types of surfacatants reaction temperature: $65^{\circ} \mathrm{C}$; concentrations of surfactants: $10 \mathrm{cmc}$

反应的速率, 即发生了胶束催化作用, 故在临界胶束 浓度处, 聚苯乙烯氯甲基化程度发生了突变; 此后随 表面活性剂浓度的增大, 胶束数量增多, 使聚苯乙烯 的增溶量继续增大, 故反应速率进一步加快, 聚苯乙 烯氯甲基化程度增大.

图 4 为对比实验的结果. 不加表面活性剂时, 反 应速率很慢, $4 \mathrm{~h}$ 后, 聚苯乙烯的氯甲基化程度仅达 $3.2 \%$, 而使用表面活性剂后(浓度均为 $10 \mathrm{cmc}$ ), 聚 苯乙烯的氯甲基化反应的速率都明显加快. 三种表 面活性剂相比, 阴离子型表面活性剂 SDS 的胶束催 化效果最差; 非离子型表面活性剂 NP-10 的胶束催 化效果居中; 阳离子型表面活性剂 CTAB 的胶束催 化效果最好, 在浓度为 $10 \mathrm{cmc}\left(3.35 \mathrm{~g} \cdot \mathrm{L}^{-1}\right)$ 条件下, 4 $\mathrm{h}$ 后, 聚苯乙烯的氯甲基化程度达 $33.1 \%$, 而且催化 剂浓度很小, 仅为 $3.35 \mathrm{~g} \cdot \mathrm{L}^{-1}$, 充分显示出胶束催化 的高效率特性.

三种表面活性剂胶束催化效果不同, 充分反映 出聚苯乙烯氯甲基化反应特定的机理. 聚苯乙烯与
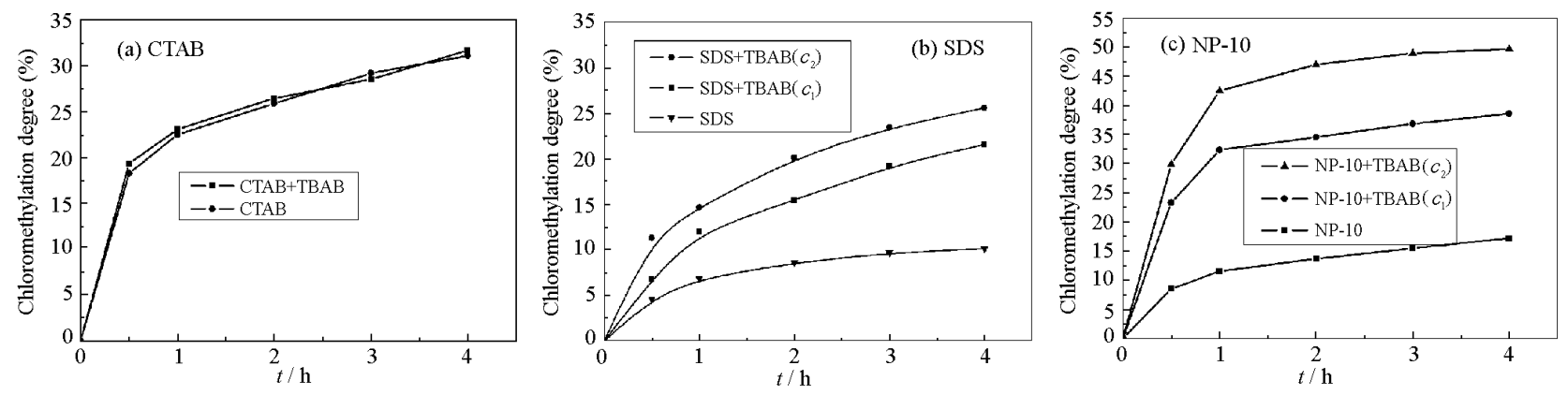

图 5 外加有机电解质对三种不同胶束催化体系中聚苯乙烯氯甲基化反应的影响

Fig.5 Effect of adding organic electrolyte on chloromethylation of PSt in three different micellar catalysis systems

reaction temperature: $65{ }^{\circ} \mathrm{C}$; concentrations of surfactants : $10 \mathrm{cmc} ; c_{1}=0.01 \mathrm{~mol} \cdot \mathrm{L}^{-1}, c_{2}=0.03 \mathrm{~mol} \cdot \mathrm{L}^{-1}$ 
在胶束催化体系中加人有机电解质 $\mathrm{TBAB}$, 考 察了其对使用三种不同类型表面活性剂的胶束催 化作用的影响规律, 实验结果示于图 5 .

从图 5 清楚地看到, 有机电解质 $\mathrm{TBAB}$ 的加人, 对使用不同类型表面活性剂的胶束催化体系具有 迥然不同的影响. 在 CTAB 体系中, TBAB 的加人 对聚苯乙烯氯甲基化反应的速率几乎不产生作用; 在 SDS 体系中, TBAB 的加人明显加快了聚苯乙烯 氯甲基化反应的速率; 在 NP-10 体系中, TBAB 的 加人, 大幅度地加快了聚苯乙烯氯甲基化反应的速 率, 加人 $0.03 \mathrm{~mol} \cdot \mathrm{L}^{-1}$ 的 TBAB, $4 \mathrm{~h}$ 后, 聚苯乙烯的 氯甲基化程度达 $50 \%$, 远远超过了 $\mathrm{CTAB}$ 体系中的 速率. 如 2.2 节分析, 有机电解质 TBAB 的加人, 会 明显增强离子型表面活性剂胶束溶液对聚苯乙烯 的增溶能力, 可能会使聚苯乙烯氯甲基化反应的速 率得以加快, 但为什么表面活性剂的类型不同, 对 胶束催化的影响截然不同呢? 显然胶束溶液增溶性 能的改变不是主要原因, 需要从胶束催化机理进行 分析. 如 2.1 节分析, 在 SDS 体系中, 在静电吸引与 疏水相互作用共同作用下, TBAB 的有机正离子 $(\mathrm{Bu})_{4} \mathrm{~N}^{+}$会嵌人阴离子头基之间, 这就会在一定程度 上减缓阴离子头基对氯负离子亲核取代反应的禁 阻作用, 故使聚苯乙烯氯甲基化反应的速率得以加 快; 在 NP-10 体系中, 在疏水相互作用下, $(\mathrm{Bu})_{4} \mathrm{~N}^{+}$亦 会结合在胶束表面, 甚至嵌人胶束之中, 使胶束表 面呈现正电性, 必然会强烈吸引氯负离子, 大大促 进亲核取代反应, 再加之 NP-10 胶束对聚苯乙烯的 增溶能力很强, 两种作用的协同, 使聚苯乙烯氯甲 基化反应的速率大幅度提高, 甚至高于 CTAB (与 Battal 等人 ${ }^{[15]}$ 研究的胶束催化合成烷基苯基醚过程 中的现象有相似之处); 而在 CTAB 体系中, 由于 静电排斥, 正离子 $(\mathrm{Bu})_{4} \mathrm{~N}^{+}$不能接近胶束表面, 水 介质中的 $(\mathrm{Bu})_{4} \mathrm{~N}^{+}$对胶束/水介质界面上的亲核取代 反应不会产生作用, 故 $\mathrm{TBAB}$ 的加人对聚苯乙烯氯 甲基化反应几乎不发生影响.

\section{3 结 论}

类似于小分子的有机反应, 线性大分子聚苯乙 烯的氯甲基反应, 在一定条件下同样可采用胶束催 化的手段, 得以高效快速地实现, 且其反应机理中 亲核取代反应为控制步骤; 在胶束催化体系中加人 有机电解质四丁基溴化铵, 在疏水相互作用下, 其 正离子 $(\mathrm{Bu})_{4} \mathrm{~N}^{+}$会结合在非离子表面活性剂胶束的
表面, 显著促进亲核取代反应, 加快氯甲基反应速 率; 在静电吸引与疏水相互作用的协同下, 正离子 $(\mathrm{Bu})_{4} \mathrm{~N}^{+}$会嵌人阴离子表面活性剂胶束的头基之间, 明显减缓阴离子头基对亲核取代反应的禁阻作用. 显然, 有机电解质在胶束催化体系中发挥着重要的 作用, 需要进一步探索研究.

\section{References}

1 Yamashita, K.; Chiba, M.; Ishida, H.; Ohkubo, K. J. Chem. Soc., Perkin Trans., 1991, $2: 367$

2 Siswanto, C.; Battal, T.; Schuss, O. E.; Rathman, J. F. Langmuir, 1997, 13: 6047

3 Mou, L. J.; Jiang, W. W.; Xia, S. L. Chinese Journal of Applied Chemistry, 2000, 17(5):563 [牟莉娟, 蒋文伟, 夏素兰. 应用化 学(Yingyong Huaxue), 2000, 17(5): 563]

4 Ouarti, N.; Marques, A.; Blagoeva, I.; Ruasse, M. F. Langmuir, 2000, 16:2157

5 van den Broeke, L. J. P.; de Bruijn, V. G.; Heijinen, J. H. M. Int. Eng. Chem. Res., 2001, 40(23):5240

6 Diego-Castro, M. J.; Hailes, H. Chem. Commun., 1998, 15: 1549

7 De Souza, E. F.; Ionescu, L. G. Colloids and Surfaces A, 1999, 149:609

8 Shen, J. J.; Zhao, Z. G.; Ma, J. M. Chemical Journal of Chinese Universities, 1997, 18(9) : 1527 [沈吉静, 赵振国,马季铭. 高等 学校化学学报 (Gaodeng Хиехiao Ниахие Хиеbаo), 1997, 18(9): 1527]

9 Stoeckel, N.; Wieland, P. C.; Nuyken, O. Polymer Bulletin, 2002, $49: 243$

10 Kavakli, C.; Özvatan, N.; Tuncel, S. A.; Salih, B. Analytica Chimica Acta, 2002, $464: 313$

11 Unsal, E.; Bahar, T.; Tuncel, M.; Tuncel, A. Journal of Chromatography A, 2000, 898:167

12 Liu, Q. F.; Gao, B. J.; Yang, Y. F.; An, F. Q. Chemical Journal of Chinese Universities, (accepted) [刘启发, 高保娇, 杨云峰,安富 强. 高等学校化学学报 (Gaodeng Xuexiao Huaxue Xuebao), 已录 用]

13 Zhu, B. Y.; Zhao, Z. G. Foundation of interface chemistry. Beijing: Chemical Engineering Press, 1996:84-93［朱埗瑶, 赵振国. 界面 化学基础. 北京:化工出版社, 1996:84-93]

14 Zhao, J. X. Journal of Fuzhou University (Natural Science), 1998, 4:91 [赵剑曦. 福州大学学报(自然科学版)(Fuzhou Daxue Xuebao(Ziran Kexue Ban)), 1998, 4: 91]

15 Battal, T.; Siswanto, C.; Rathman, J. F. Langmuir, 1997, 13: 6053 